\title{
Aluminum Clad Spent Nuclear Fuel Task 5: Oxide Layer Response to Drying Experiment Test Plan
}

\author{
Rebecca E Smith
}

August 2019

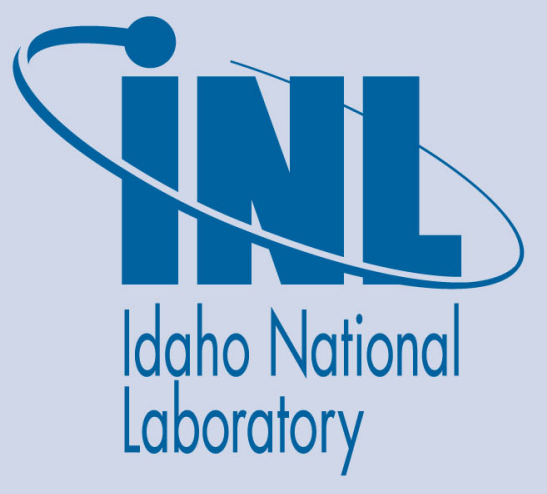

The INL is a U.S. Department of Energy National Laboratory operated by Battelle Energy Alliance 


\title{
Aluminum Clad Spent Nuclear Fuel Task 5: Oxide Layer Response to Drying Experiment Test Plan
}

\author{
Rebecca E Smith
}

August 2019

Idaho National Laboratory Idaho Falls, Idaho 83415

http://www.inl.gov

Prepared for the U.S. Department of Energy Office of Environmental Management Under DOE Idaho Operations Office Contract DE-AC07-05ID14517 
Prepared by:

Nown 5 dincti-

Rebecca Smith

INL Principal Investigator

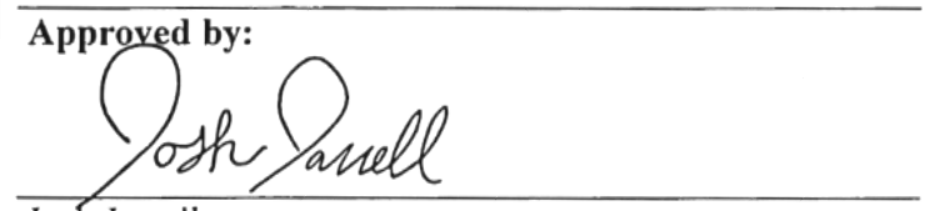

Josh Jarrell

INL Integration Lead

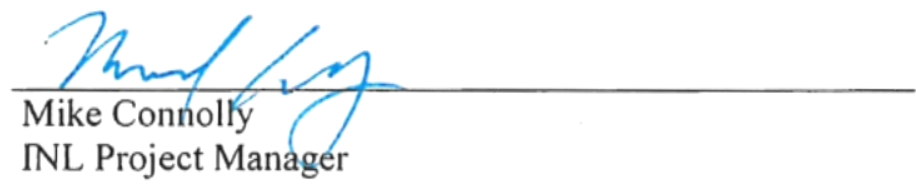

$5 / 17 / 2019$

Date

$\frac{5 / 17 / 2019}{\text { Date }}$

05/29/2019

Date

$5 / 22 / 2019$ 
ASNF Task 5 Experiment Test Plan

Revision: 1

Effective Date: 8/15/2019

\section{REVISION LOG}

\begin{tabular}{|c|c|c|c|}
\hline Rev. & Date & Affected Pages & Revision Description \\
\hline 1 & $8 / 15 / 2019$ & $14,15,17, \& 18$ & Updates QA references and schedule assumptions \\
\hline & & & \\
\hline & & & \\
\hline & & & \\
\hline & & & \\
\hline & & & \\
\hline & & & \\
\hline & & & \\
\hline & & & \\
\hline & & & \\
\hline & & & \\
\hline & & & \\
\hline & & & \\
\hline
\end{tabular}




\section{SUMMARY}

This document presents a test plan developed to assess the fundamental effectiveness of two widely accepted processes for drying spent nuclear fuel: vacuum drying and helium forced-gas drying. The experimental activities planned for Task 5 follow from the "Aluminum Clad Spent Nuclear Fuel Long Term Dry Storage Technical Issues Action Plan.'[1] The objectives are to evaluate the response of the oxide corrosion layer on aluminum clad spent nuclear fuel (ASNF), processed at temperatures above $100^{\circ} \mathrm{C}$, to understand and compare the behavior of oxide corrosion layer compositions resulting from a range of credible procedural variations during the transition of ASNF from wet storage to dry storage. 


\section{CONTENTS}

SUMMARY iii

ACRONYMS $\mathrm{V}$

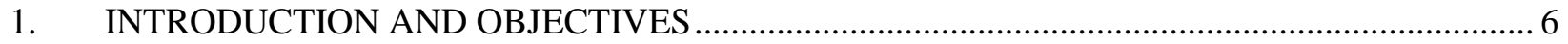

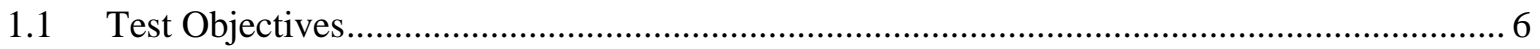

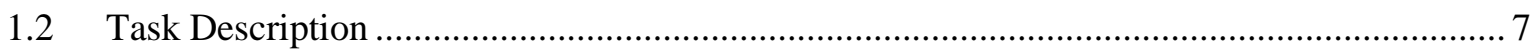

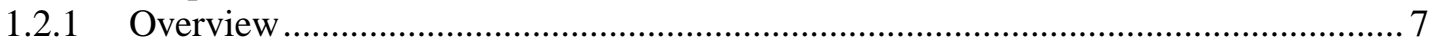

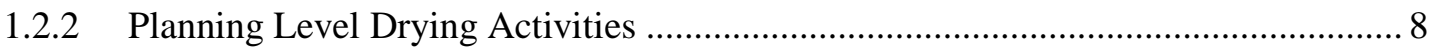

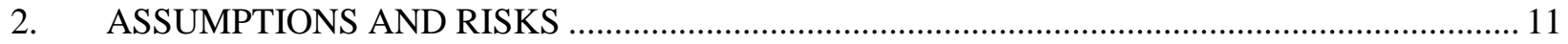

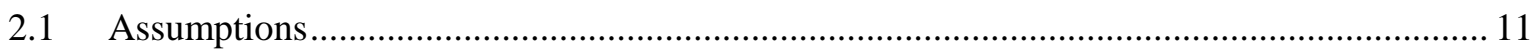

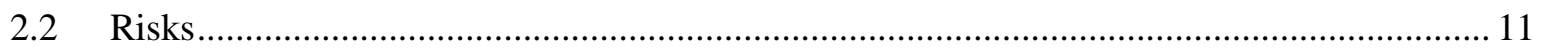

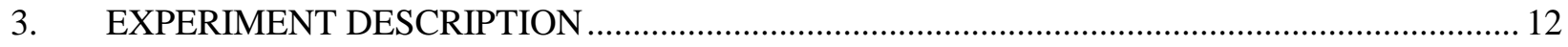

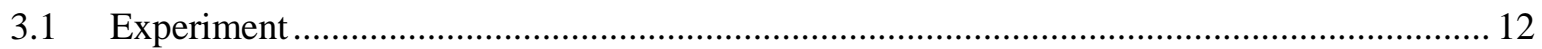

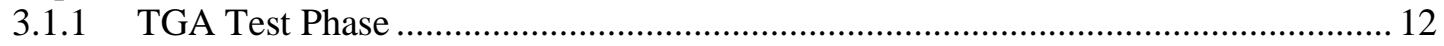

3.1.2 Small Chamber Test Phase …............................................................................ 12

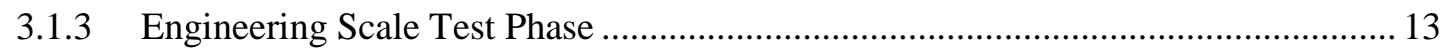

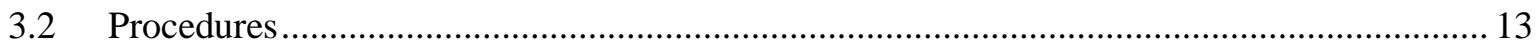

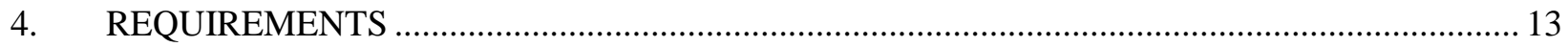

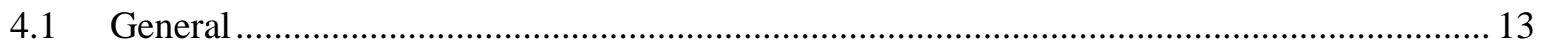

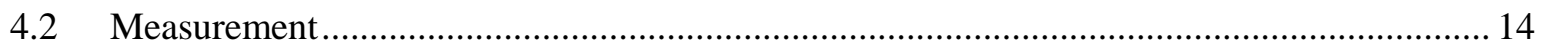

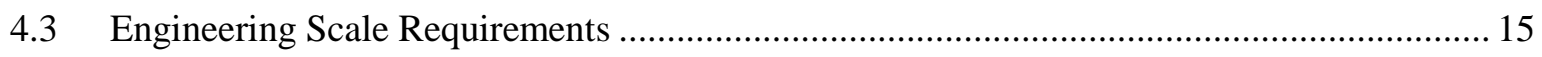

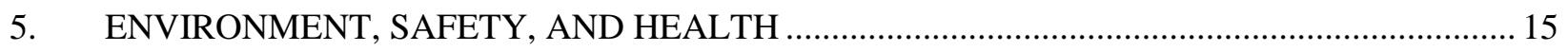

6. QUALITY ASSURANCE AND DATA/RECORD MANAGEMENT ..................................... 15

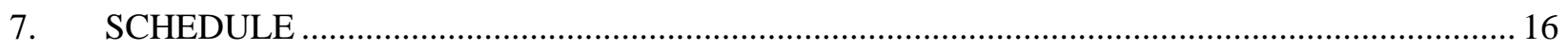

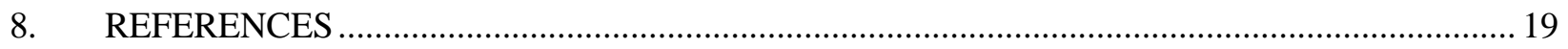




\section{FIGURES}

Figure 1. Schematic Timeline for Deliverables (and supporting activities)

\section{TABLES}

Table 1. Planning Level Activities, Leads, and Participants

Table 2. Scheduling Assumptions

\section{ACRONYMS}

ASNF aluminum clad spent nuclear fuel

DTA differential thermal analysis

INL Idaho National Laboratory

LI laboratory instruction

SEM scanning electron microscope

SNF spent nuclear fuel

SRNL Savannah River National Laboratory

TGA thermogravimetric analyzer

USC University of South Carolina

XPS X-ray photoelectron spectroscopy

XRD $\quad \mathrm{X}$-ray diffraction 


\section{ASNF Task 5 Experiment Test Plan}

Revision: 1

Effective Date: 8/15/2019

\section{INTRODUCTION AND OBJECTIVES}

Task 5 focusses on the factors expected to influence the effectiveness of a drying procedure in the removal of water generally from aluminum-clad spent nuclear fuel (ASNF), and specifically in the release of chemically bound water from oxide corrosion layers and any subsequent influence on the oxide (and alloy) morphology. The process temperatures and composition of the oxide layers relevant to the Task 5 experimental plan will be informed by the investigations under Task 1: Oxyhydroxide Layer Behavior and Chemistry at Idaho National Laboratory (INL), as well as recent work at Savannah River National Laboratory (SRNL).

University of South Carolina (USC) has contributed to the development of this test plan through INL Contract \#207046. Holtec International has contributed to the development of this test plan through INL Contract \#215725. USC and Holtec concurrence with this plan is inherent to the revised or new contract agreements and scopes of work formalized through INL Procurement.

\subsection{Test Objectives}

Task 5 examines the influence of the drying process on the chemical composition of the corrosion layer associated with the aluminum SNF cladding: the environmental exposures (thermal excursions, durations, and modes of mass transfer for removal of water) integral to the transfer of ASNF from the reactor pool or other wet storage configuration to dry storage. Task 5 considers both the incumbent drying rates and the extent of transition in aluminum oxide morphology in this context. This effort aspires to address the knowledge gap between Task 1: Oxyhydroxide Layer Behavior and Chemistry and Task 4: Performance of ASNF in Dry Storage, by acquiring data to compare the efficacy of procedural variations in drying methodologies. Specifically this work seeks to identify the optimal drying process and processing conditions to ensure minimal residual moisture without initiating or propagating mechanisms likely to be detrimental to the long term performance of the ASNF in dry storage.

The specific goals of Task 5 are, for both vacuum drying and helium forced-gas drying processes:

1) Develop and validate Drying Model for integration into ASNF Dry Storage Model. Assess residence time and other constraints necessary to achieve a relatively uniform temperature of $200^{\circ} \mathrm{C}$ across and through all plates without exceeding $250^{\circ} \mathrm{C}$.

2) Assess the extent to which the drying process parameters (stage duration, pressure and gas flow rate, number of stages, maximum temperature and nominal thermal profile across the load) may influence the relative composition and morphology after removal of a quantity of liquid water.

3) Evaluate the applicable expenses (equipment purchase, installation and maintenance, operating costs, ASNF throughput, and practical constraints).

4) Consider process endpoint-determination reliability in the context of resultant relative composition and morphology and uncertainties in moisture and thermal characteristics of initial ASNF load.

5) Identify any pinch-points, capacity limitations, or operational factors that favor or impede the efficacy of either process. 


\section{ASNF Task 5 Experiment Test Plan}

Revision: 1

Effective Date: 8/15/2019

\subsection{Task Description}

\subsubsection{Overview}

In the interest of investigating the behavior of aluminum oxide corrosion layers as a result of the drying process employed, surrogate materials (prepared by SRNL or by INL or by others under INL or SRNL direction) will be used. Four factors are relevant: material composition of the corroded surfaces retrieved wet, process temperature, process gas environment, and conditional procedural operations (durations of process steps). Each of these factors contributes to the Task 5 test matrix, as informed or constrained by practical considerations.

Task 5 ASNF Drying Experiment requires two types of surrogate material: corrosion chemistry surrogate and fuel geometry surrogate. The near term objectives of the TGA and Bench scale scoping studies are to support the Engineering scale drying tests. Specifically, these scoping studies pertain to the corrosion chemistry. The intent is to validate the comparison of loose powder (scrapings) to surface corrosion with respect to performance during drying, to assess the quantities of corrosion chemistry surrogate necessary for the Engineering scale experiments, to identify a corrosion protocol that provides uniform and sufficient quantities of surface corrosion on surrogate material to support the test matrix, and to down select to a single alloy for surrogate surface corrosion development. Fabrication of fuel geometry surrogate is expected to proceed in parallel, subject to the Simulation Strategy and acceptance criteria to be defined in the Engineering Scale Experiment Design.

Given the limited range of practical and allowable ASNF drying conditions (temperature, gas flow, pressure, process duration), the representative case (a thin to moderate corrosion layer dominated by Boehmite) is unlikely to exhibit discernable difference in drying performance upon exposure to the two competing drying processes (heated vacuum drying and helium forced gas dehydration) regardless of the drying test parameters. The worst case for corrosion-borne water (significant quantities of Gibbsite and/or Bayerite as well as Boehmite) is both difficult to replicate consistently and time consuming to produce at all. However, this worst case surrogate is necessary to provide the relevant drying process evaluation (determine which process conditions are most effective at removing water bound to the corrosion layer).

For ASNF Drying purposes, down selection to a single alloy has been recommended on the following basis.

Alloys AA 1100, AA 5052, and AA 6061 are all resident in the DOE ASNF inventory, but testing all three both expands the test matrix and adds a variable to the effort of replicating sufficient quantities of suitable surrogate. Under most conditions, AA 1100 is slightly more vulnerable to corrosion, whereas AA 6061 is representative of a somewhat larger fraction of the ASNF inventory. For the purposes of Drying, the quantity and morphology of oxide is deemed to be of significantly more importance than the cladding alloy content, because the chemisorbed moisture that drying is intended to remove is associated with the surface oxide layer not the alloy per se.

However, thermodynamic analysis indicates AA 6061 and to a lesser extent AA 5052 may be susceptible to alloy transformations upon exposure to relatively low temperatures $\left(100^{\circ} \mathrm{C}\right.$ to $\left.250^{\circ} \mathrm{C}\right)$ during drying, whereas AA 1100 exhibits nearly ideal behavior with only limited phase changes below $400^{\circ}$ C.[2] Use of AA 6061 for (Task 5) ASNF Drying experiments offers an opportunity to examine the extent of both alloy and corrosion layer performance during drying.

The differences in corrosion rates and distribution of (Gibbsite/Bayerite/Boehmite) corrosion products among these three alloys are relatively small, depending much more heavily on environmental factors such as temperature, $\mathrm{pH}$, and water quality than the specific aluminum alloy.[3-8] 


\section{ASNF Task 5 Experiment Test Plan}

Revision: 1

Effective Date: 8/15/2019

Therefore, the choice has been made to down select immediately, without further small scale testing on multiple alloys, and use AA 6061 exclusively for the purposes of TGA, small chamber, and engineering scale Task 5 Drying activities.

Initial and eventual material composition (before and after each drying test) will be evaluated with $\mathrm{x}$-ray diffraction (XRD) and x-ray photoelectron spectroscopy (XPS) as appropriate to assess the baseline oxide (and alloy) morphology and correlate any observed composition changes with details of specific drying procedures or material origin. Other analyses, for example examination of specimens using scanning electron microscope (SEM), will be employed as needed for comparison of performance.

Maximum temperature and thermal profile, the height and duration of elevated temperature during the drying process will be varied to provide meaningful comparison between the vacuum drying and helium forced-gas drying processes as well as to explore the consequences to oxide (and alloy) morphology under the nominal surface conditions of a high temperature (short term thermal excursion) inherent to any SNF drying environment.

Gas composition and flow or pressure profile during drying (heat transfer, concentration gradient or evacuation driven mass transfer, determination of process endpoint) will not be directly comparable between the two major drying techniques. As time allows, a minimal range of optimal parameters will be estimated for each technique.

Develop (unit operations) heat and mass transfer equipment and operating cost schemes for each drying process and examine performance with scale normalized by the same nominal material load. Conditional limits (timeframe, practical constraints on SNF handling, credible process operations given diminishing benefit for the cost) for ASNF and for test environments will be considered and their influence on experimental design decisions documented. SNF handling operational constraints tend to limit process drying time to a few days, with strong preference to completion of the process operation within a single work shift. Meaningful test parameters may be influenced by analytical detection limits, and by timely results of the other ASNF Dry Storage Project tasks. Test temperatures and pressures may be limited by the capacities of readily available instruments and equipment.

The heat and mass transfer schemes will be revised to accommodate variations in scale and evaluate the results against observations of actual ASNF from Task 4 and the dry storage model of Task 3. Results of Task 2 in conjunction with Task 3 should illuminate the adequacy of the drying process for the specific dry storage configuration (vented or sealed). Drying models for each process will be developed to correlate results to process variables and to interface with the (sealed and vented) ASNF dry storage models being developed under Task 3 .

\subsubsection{Planning Level Drying Activities}

The Overall Task 5 Drying Activity Lead is INL/Rebecca Smith. Primary Engagement involves University of South Carolina (USC)/Travis Knight, Holtec/John Griffiths, SRNL/Bob Sindelar, INL/Josh Jarrell and Mike Connolly. High Level Schedule is based on this task being planned for 1 year.

Primary participants (Mike Connolly, INL, Travis Knight, USC, and John Griffiths, Holtec, or their designated delegates) have concurrence authority at identified hold points to confirm completion of requisite precursor activities and adequacy of engagement for subsequent project success. Other participants, engaged for their relevant expertise, are responsible to advise the primary participants and facilitate collaboration. Activities, Leads, and Participants are outlined in Table 1. 
Table 1. Planning Level Activities, Leads, and Participants

1.) ASNF Drying (Task 5) Planning Documents, Drying Activity Lead - INL/Rebecca Smith, Document-specific leads with all primary participant engagement

a. Requirements Document (to be integral to Test Plan): Lead - INL/Rebecca Smith, (completed 1/15/2019, included in ASNF Task 5 Experiment Test Plan under Section 4)

b. Experiment Design Document (to be identified in Test Plan, but issued separately): Lead USC/Travis Knight, (3 weeks, concurrent with Activity 2, below)

c. Deliverable Planning Timeline (included in ASNF Task 5 Experiment Test Plan under Section 7, to be updated and maintained as needed through project control and subcontract management): Lead - INL/Rebecca Smith, (draft released with 2/18/2019 conference call agenda, initial issue with ASNF Task 5 Experiment Test Plan)

d. ASNF Task 5 Experiment Test Plan - this document.

Hold point for ASNF Task 5 Experiment Test Plan issue, Lead - INL/Rebecca Smith.

2.) Engineering Scale Experiment Design, Lead - USC/Travis Knight, (12 weeks, pending concurrence on this ASNF Task 5 Experiment Test Plan)

a. Develop Drying Process Models (for vacuum drying and for helium forced gas drying) building upon the ASNF Dry Storage Models (Task 3) Also engage INL/Alex Abboud

b. Establish Simulation Strategy including fabrication acceptance criteria (coordinate the materials and their configuration to address Requirements) Also engage Holtec/John Griffiths INL/Tedd Lister, and SRNL/Bob Sindelar

c. Confirm process and in situ instrumentation accommodates experimental design and test parameters for model validation Also engage Holtec/John Griffiths

d. Identify Analytical Capabilities, Instruments, and Logistics necessary for sample transport and analysis, Also engage SRNL/Dave Herman, and INL/Phil Winston

e. Use small chamber testing to support design decisions and demonstrate replication and/or limitations of TGA phase analyses

f. Planning Chronology of Experimental Tests to satisfy Requirements (discreet from documentation of the design, correlate design choices and experiment matrix to requirements and objectives)

Hold point for Engineering Scale Experiment Design Document issue, Lead - USC/Travis Knight.

3.) Fabrication and Installation of Engineering Scale Test Systems Lead - Holtec/John Griffiths, (12 weeks, pending approval of Engineering Scale Experiment Design Document)

a. Simulate Standard Canister with Type 1a Basket configuration (for a nominal 5 foot tall single basket section)

b. Provide mock ASNF plate fuel element basket loading configuration (10 elements, 15 to 20 plates per element, ATR dimensions or similar), engage USC/Travis Knight and others regarding Simulation Strategy as described in Engineering Scale Experiment Design

c. Develop aluminum oxyhydroxide surface corrosion for chemical analysis of drying performance, engage Travis Knight and others regarding Simulation Strategy 


\section{ASNF Task 5 Experiment Test Plan}

Revision: 1

Effective Date: 8/15/2019

d. Establish (or confirm) process control equipment and feedback instrumentation (including endpoint determination) for both vacuum drying and helium forced gas drying processes, engage USC/Travis Knight regarding experimental tests and data needs

e. Acceptance of fabricated items to be consistent with Simulation Strategy identified in Engineering Scale Experiment Design Document

Hold point for acceptance of fabricated items, Lead - Holtec/John Griffiths, Lead for Chemistry and ASNF Geometry Surrogates - INL/Rebecca Smith.

4.) System Operability Testing of Engineering Scale Systems (including documentation of functional testing results, issues, and issue resolutions) Lead - Holtec/John Griffiths, (5 weeks, pending finalization of Fabrication and Installation)

Hold point for acceptance of Operability Test approach and criteria for success, Lead Holtec/John Griffiths.

a. Vacuum Drying Functions (heat input, vessel evacuation, purge flow, vessel isolation and pressure response, thermal response within basket and surrogate ASNF geometry)

b. Helium Forced Gas Drying Functions (heat input, helium supply and control, outlet gas moisture, thermal response within basket and surrogate ASNF geometry)

c. Sample Handling and Analysis of chemistry surrogate in coordination with USC (packaging and transportation of samples, experimental control, sample preparation and analysis protocol)

Hold point for concurrence on successful Operability Test completion, Lead - Holtec/John Griffiths.

5.) Experiment (including documentation of test protocols, experimental observations, recorded data, results, analyses, and conclusions) Lead - USC/Travis Knight, in coordination with Holtec, (20 weeks, pending approved completion of Operability Testing)

a. Limited Vacuum Drying Optimization

b. Limited Helium Forced Gas Drying Optimization

Hold point for transmittal of optimization data, Lead - USC/Travis Knight.

c. Vacuum Drying Experimental Tests

d. Forced Gas Drying Experimental Tests

e. Sample Analysis to indicate process performance for ASNF

f. Model Validation and update to models as needed

Hold point for transmittal of experimental test data and analytical results, integration of Drying Models into Dry Storage Models, Lead - USC/Travis Knight.

6.) Final Project Report Overall Lead - Rebecca Smith, (6 weeks)

a. Discussion of Drying Model (Lead - USC/Travis Knight)

b. Description of Equipment and Materials (Lead - Holtec/John Griffiths)

c. Experiment Data, Results, and Analyses (Lead - USC/Travis Knight)

d. Conclusions

Hold point for agreement on any limitations on final report distribution, Lead - Rebecca Smith. 


\section{ASNF Task 5 Experiment Test Plan}

Revision: 1

Effective Date: 8/15/2019

\section{ASSUMPTIONS AND RISKS \\ 2.1 Assumptions}

The primary assumption for Task 5 is that materials received or cultivated for use will be both representative and sufficient in quantity to enable discrimination among the variable drying process parameters being tested. Historical "outliers", ASNF which has experienced unusual treatment during service, will not be specifically considered in this study. Such historical events could be poor chemical control in service or wet storage, physical damage from moving, out of normal operation, exposure to biological or soil debris. This could create a myriad of potential chemical environments that are difficult to recreate in a testing environment. Recreating such conditions is outside of the scope of this test plan, albeit something that could be examined in the future, and could provide a measure of the relative robustness of a drying process selected.

The test plan assumes that the drying process parameter variables selected (temperatures, cover gas conditions, procedural durations, and oxide moisture loads) are comparably optimized for the respective vacuum drying and helium forced-gas drying procedures. These considerations can vary significantly with process scale, but must be limited to fit the funded scope and performance period allotted.

Predicting relative process efficiencies at full scale is the primary goal of Task 5 . This test plan aims to correlate process conditions during drying to qualitative and semi-quantitative information about the resultant oxide morphology. This test plan will benchmark these values against the principle reactions, trends, and observations identified in Tasks 1-4.

\subsection{Risks}

The data collected in this exercise serve the purpose of providing information where little to none exists: SNF drying procedures were developed to remove liquid water, relying upon the dry storage configuration and neglecting the chemistry of the oxide over the long term. To this point, analyses have made conservative assumptions about the reaction with water, including that tightly bound as an oxyhydroxide. The test plan and subsequent use of data will inform future decisions for drying process selection in the context of the intended SNF storage configuration and presumed final disposition.

An important risk to use of data from the test plan is to assume that all chemical reactions are considered in this test plan. Importantly, radiolysis is covered as a separate task, where relatively benign water could become more reactive by radiolytic activation. Thus results of Task 1 and Task 2 activities must be considered as a whole in assessing the chemical stability of ASNF. In conjunction with Task 3, these activities will substantiate the adequacy or limitations of conventional SNF drying for the subsequent long term dry storage of ASNF.

There will always be a risk in performing laboratory experiments in order to assess real world conditions. It is simply impossible to exactly simulate the drying procedures ASNF has experienced. The test plan has taken the approach in attempting to use realistically produced specimens as a starting point. The conditions developed for the test matrix come from best assessments provided by Tasks 1 through 4 . Likewise, operational drying protocol and realistic endpoint determination will generally necessitate the introduction of at least some liquid water to emulate the overall system response.

Given limited time and resources, this test plan considers the effects of only a limited set of benchmark process variables - anticipating similar constraints on optimization stepping up in scale from TGA to bench top apparatus to engineering test facility. There is the additional risk that these variations in process scale may be insufficient to discriminate meaningful distinctions in the relative efficiencies of full 


\section{ASNF Task 5 Experiment Test Plan}

Revision: 1

Effective Date: 8/15/2019

scale drying processes. However, the Task 5 test matrix will at least provide a controlled set of data to facilitate such a comparison.

\section{EXPERIMENT DESCRIPTION \\ 3.1 Experiment}

These drying activities are organized in a three tiered approach, specifically considering issues associated with change in scale, with the first tier employing thermogravimetric analysis of small (analytical scale) quantities of corrosion product, the second tier examining small chamber performance of corroded coupons (bench scale), and the third tier using mock fuel assemblies in an (engineering scale) instrumented test facility. The scope of test environments is bounded by practical restrictions on the handling of ASNF, with functional durations generally limited to protocol completed within a single work shift, consistent with historic drying practices employed at INTEC.

The TGA and small chamber scoping tests have two main objectives:

- Examine / illuminate any variations between performance of the corrosion on the alloy surface and performance of loose powder (scrapings), and

- Consider implications for the development of surrogate materials (what quantity of surrogate is sufficient, and how can it be developed rapidly and with consistent composition).

These scoping study objectives may be obviated by research progress on other ASNF Long Term Storage tasks.

Design of the Engineering Scale Experiment will be developed in parallel with the TGA and bench scale testing and will be predicated on the development of drying process models. The Engineering Scale Experiment Design Document will define the ASNF simulation strategy, establish test parameters for drying process model validation, and plan the chronology for engineering scale experiments and analyses. USC will prepare this design document for Holtec concurrence and INL approval.

Operability Test Plans will be prepared by Holtec for USC concurrence and INL approval. Similarly Holtec will document results of operability tests for USC concurrence and INL approval of successful operability test completion.

\subsubsection{TGA Test Phase}

Look at the drying performance of powder samples in the TGA, evaluate the relative performance of vacuum and helium carrier gas drying of these powder samples. The TGA test sequence will develop a baseline for initial comparison of corrosion samples developed from the aluminum alloy AA 6061 . Evacuation cycles (for vacuum drying) and helium conditions (for forced-gas drying) will be optimized for one or more nominal TGA loads to test sensitivity to initial water burden, thermal profile and nominal mass (from each alloy source). Powder samples will be interrogated at temperatures from $100^{\circ} \mathrm{C}$ to $250^{\circ} \mathrm{C}$ within the context of the drying process at instrument scale. These small scale parametric TGA tests may be sufficient to indicate whether the differences in morphology and composition among the three aluminum alloy variations are significant to process optimization. Subsequent TGA analysis with exposure above $250^{\circ} \mathrm{C}$ can provide a quantitative measure of residual chemisorbed water.

\subsubsection{Small Chamber Test Phase}

Scale up to drying individual prepared solid specimens (coupons) using a tube furnace or an adaptation of the Task 1 apparatus to achieve the desired drying process protocol. Performance of the 
small chamber will be benchmarked to consider thermal inertia and the impact of boundary conditions with the increase in capacity. Process optimization for the larger material load will be informed by TGA results.

\subsubsection{Engineering Scale Test Phase}

Facility modification will be needed to provide full instrumentation to a proportionate cross-section of full length mock ASNF. Performance of large simulated ASNF pieces will be benchmarked against the corroded aluminum coupons (as used in small chamber) with the overall simulation strategy to be developed in conjunction with the Engineering Scale Experiment Design. Baseline effects of adsorbed water within the piping and internal surfaces of the large drying system will be considered in the context of the residual moisture affiliated with the corrosion layer on ASNF. Verification of optimal process parameters may be performed with optical emission spectroscopy (OES). Heated mock ASNF element and judiciously located thermocouples and/or FLIR IR Camera or other means of temperature monitoring will facilitate thermal model validation and drying model development.

\subsection{Procedures}

Performing experiments requires careful entry of all relevant information into the lab notebook (electronic or hard copy). This includes recording experimental steps, specimen identification, calibration information, experimental time and date. Electronic data acquisition filenames are to be traceable to the specific test environment with cross referencing to acknowledge both local clock time and any automated run times. If a specific set of tasks are to be performed in repetition, a copy of that procedure can be pasted in the notebook and referred to. The important information from each experiment and or specimen needs to be clearly identified both to be traceable to the relevant analyses and in order for an independent person to understand what was done.

Specimens will have unique identifier (or ID\#), either from the specimen provider or applied by user before starting the experiment. This will allow each specimen to be tracked, particularly when a parallel operation such as specimen pre-treatment is performed. Also, the identifier, the location of origin, and the sampling and preparation methodology will be noted when powder samples are taken or when larger items (such as full length surrogate ASNF) must be cut to size for analysis. These marks will be recorded in notebook for each procedure followed.

\section{REQUIREMENTS}

\subsection{General}

Both vacuum drying and helium forced gas drying are to be modeled and experimentally tested to provide a documented comparison of performance for use ASNF.

The objectives are to evaluate the response of the oxide corrosion layer on ASNF, processed at temperatures above $100^{\circ} \mathrm{C}$, to understand and compare the behavior of oxide corrosion layer compositions resulting from a range of credible procedural variations during the transition of ASNF from wet storage to dry storage. This will require evaluation of both the incumbent drying rates and the extent of transition in aluminum oxide morphology. The mechanisms for process endpoint determination may also be significant to the quantitative achievement of success. TGA and small chamber testing are anticipated to demonstrate relevant variations and uncertainties inherent to surrogate material selection and preparation.

Safe work practices will be followed at all of the facilities supporting ASNF drying experiments. Activities to be performed at Idaho National Laboratory will be authorized under conduct of research 


\section{ASNF Task 5 Experiment Test Plan}

Revision: 1

Effective Date: 8/15/2019

documented in Laboratory Instructions (LI). Additional approval may be required for compliance with facility-specific conduct of operations. Quality assurance associated with these ASNF drying and analysis activities will comply with all applicable requirements set forth in the INL Quality Assurance Program based on ASME NQA-1 2008/09a.

Lab notebooks will be used to record all pertinent information including (but not limited to) calibrations numbers and due dates, sample identification numbers and numbering practice (for traceability to original alloy and treatment as well as supplier and date of receipt). Comparable records will be maintained by USC, Holtec, Savannah River National Laboratory and Idaho National Laboratory participants, for all of the supporting activities conducted at their respective facilities.

Sample materials will need to be properly packaged to preserve sample integrity between (the time and location of) experiment and analysis. Blanks will be used to establish baseline analytical performance and confirm the adequacy of sample control. After experimental testing is complete, samples will be stored individually in containers such that their integrity is preserved. Each container will be labeled such that the experimental conditions of the sample are indicated.

For those instruments and analyses outside the purview of INL Calibration Lab, including TGA, DTA, XRD, XPS, SEM, and metallography, suitable routine practices and application-specific controls will be identified, employed, and documented to facilitate the reproducibility of results and to substantiate the validity of both the testing and analytical processes. Additional analysis will be employed to benchmark the relative composition and morphology of engineering test materials against those from both the TGA and the small chamber.

All experimental activities at INL outside of maintenance will be logged in a registered INL lab notebook (electronic or hard copy). Record all pertinent information such as calibrations numbers and due dates and specimen identification numbers (traceable to original alloy and treatment as well as date of receipt from SRNL). After testing is complete, store specimens individually in containers such that their integrity is preserved. Label such that the experimental conditions are present on the container. Comparable records will be maintained by USC for all experimental testing conducted under their direction.

\subsection{Measurement}

In the experimental processes, calibrated items will be used wherever the INL Calibration Lab offers qualified services using certified standards, including laboratory balances, calipers, torque wrenches, thermocouples, mass flow and pressure measurement devices. The person performing the experiment should record calibration specific information including calibration numbers and calibration due date, and these data are fully traceable by S\&CL number through the INL Calibration Lab database.

For those instruments and analyses outside the purview of INL Calibration Lab, including TGA, DTA, XRD, XPS, SEM, and metallography, as well as experimental processes and analyses conducted at USC or Holtec, suitable routine practices and application-specific controls will be identified, employed, and documented to facilitate the reproducibility of results and to substantiate the validity of both the testing and analytical processes. Additional analysis will be employed to benchmark the relative composition and morphology of engineering test materials against those from both the TGA and the small chamber. 


\section{ASNF Task 5 Experiment Test Plan}

Revision: 1

Effective Date: 8/15/2019

\subsection{Engineering Scale Requirements}

In order to provide an appropriate representation of the heat transfer and flow path and to allow for surface area exposure relevant to aluminum oxyhydroxide chemistry during drying, the system size for engineering scale experiments will accommodate use of a nominal 5 foot tall (single basket) single tier of surrogate ASNF with the 18 inch cross sectional configuration of the DOE Standard Canister and Type 1a basket design. ASNF plate type fuels typically have 15-20 plates per fuel element. A Type 1a basket is designed to hold 10 elements, using ATR fuel as a base case.

Modeling will be employed in the detailed design of test load, including the type and configuration of surrogate materials. Experimental parameters will be selected to validate the thermal model and assess residence time (and other constraints) to achieve (a relatively uniform) temperature of 200 degrees Celsius across and through all plates without exceeding 250 degrees Celsius.

The vacuum drying and helium forced gas drying operating protocols will each need to be optimized (within reason given the scope of the project) to enable an equitable process performance comparison. This optimization is assumed to be an iterative process with potentially differing optimal operating parameters depending on details of the (water and simulated ASNF) load. Modeling work is expected to identify to the range of initial conditions likely to differentiate performance of the respective drying processes. Current INL ASNF handling practices assume the presence of liquid water before drying, allow for a heat load of up to 30 Watts per element, and prevent ASNF temperature from exceeding $250^{\circ} \mathrm{C}$.[9] This optimization effort is meant to facilitate the choice between the two drying processes where one may be more efficient or more cost effective than the other for a subset of ASNF with minimal surface corrosion. Emphasis for optimization is on the liquid water removal which has historically driven SNF drying operations, while the focus of the engineering scale experimental testing that follows is to distinguish any differences between the two drying processes in the resultant surface chemistry.

Results from TGA evaluation of powder specimens indicate that gibbsite and bayerite tend to decompose over a relatively wide range of temperatures, and therefore removal of adsorbed moisture may be incomplete or highly time sensitive for any given set of drying parameters. Establishing a satisfactory understanding of the upper bounds of the thermal performance envelope may involve testing at slightly higher test temperatures to achieve and maintain temperatures approaching $250^{\circ} \mathrm{C}$ throughout the surrogate fuel load.

\section{ENVIRONMENT, SAFETY, AND HEALTH}

Portions of the test plan will be performed at REC facilities: INL Research Center (IF-602) and the Energy Innovation Laboratory (EIL). The activities will be performed under two Laboratory Instructions (LI). Specimen pre-filming will be performed under LI-589 "Corrosion and Electrochemical Science". Corrosion testing will be performed under LI-610 "Oven and Furnace Operation". Pertinent requirements are contained within the appropriate LI. Engineering scale operations will be conducted at Holtec, with data and sample analyses coordinated with USC. Conduct of operations and conduct of research in each case will be governed by the requirements of the respective host facilities.

\section{QUALITY ASSURANCE AND DATA/RECORD MANAGEMENT}

Quality assurance activities associated with this work comply with all applicable requirements set forth in the INL Quality Assurance Program based on ASME NQA-1 2008/09a. 


\section{SCHEDULE}

Task 5 milestones and deliverables are based on discussions with INL and SRNL participants as well as cost estimates and scope information provided by USC and Holtec. A tentative schedule is presented below with milestones and deliverables outlined for planning purposes. Completion dates and the experiment test plan will proceed as governed by the TTP and by the respective contracts between INL and USC, and INL and Holtec. Scheduling assumptions are outlined in Table 2. Figure 1 shows a tentative timeline for the planned activities.

Task 5 Milestones and Deliverables:

\subsection{Milestone - Issue ASNF Task 5 Experiment Test Plan.}

5.2 Deliverable - Identify and confirm the sources and availability of items and personnel to establish the means to provide sufficient quantities of surrogate materials for both evaluation of chemisorbed water (representative of surface corrosion water retention) and for physical geometry (representative of ASNF load configuration) during drying. Availability and size constraints of controlled environment immersion vessels may otherwise become critical path problems with schedule impact. (Delivery of actual surrogate materials is addressed separately below.)

5.3 Deliverable - Develop a TGA scale and bench scale test matrix to provide suitable results based on the quantities and variations of surrogate materials being produced.

5.4 Deliverable - Evaluate the decomposition kinetics of surrogate corroded aluminum and supply the relevant information to USC:

- Receive AA 6061 coupons. Immerse under controlled conditions to optimize growth of gibbsite and bayerite (over boehmite).

- Assess handling for composition changes (to accommodate transit / time between immersion and testing.

- Establish TGA/DTA (thermogravimetric analyzer / differential thermal analysis) baseline and assess the adequacy of corrosion growth for planned tests.

5.5 Milestone - Issue Engineering Scale Experiment Design Document.

5.6 Deliverable - Develop a process spreadsheet to compare mass \& energy balance, as well as capital equipment and operating costs of vacuum and helium forced-gas processes with similar capacities (unit ops): Estimate kinetics for both processes for bench scale decomposition testing from TGA test sequence. Establish framework for drying models.

5.7 Deliverable - Conduct TGA and bench scale vacuum and helium forced-gas drying tests (nominal sample size $\sim 150 \mathrm{~g}$ ): Employ periodic weighing (pre-test, post-test, and intermittent to process as needed) to assess performance and refine process spreadsheet for significant variations with scale. Perform posttest XRD (\& XPS) \& cross-sectional metallography to evaluate resultant composition. Interim report summarizing (preliminary / TGA and bench scale) drying results as of September 3, 2019 to be issued by 9/30/2019 in support of the ASNF Technology Development Completion Report.

5.8 Milestone - Approval/acceptance of fabricated items (Type 1A basket, ASNF full length elements, and aluminum plates with surface corrosion). 


\section{ASNF Task 5 Experiment Test Plan}

Revision: 1

Effective Date: 8/15/2019

5.9 Deliverable - Approval/acceptance of System Operability test protocol and criteria for success.

5.10 Milestone - Successful completions of System Operability testing.

5.11 Deliverable - Transmittal of optimization data, experimental test data and analytical results.

5.12 Deliverable - Integration of Drying Models into Dry Storage Models.

5.13 Milestone - Issue Final Report on ASNF Drying.

Table 2. Scheduling Assumptions

\begin{tabular}{|c|c|c|c|}
\hline Position & Start Date & +1 End Date $-\uparrow$ Milestone/Activity & Expected Duration (days) \\
\hline 1 & $5 / 15 / 2019$ & 5/15/2019 Start & 0 \\
\hline 2 & $5 / 15 / 2019$ & 5/29/2019 'Edit ASNF (Task 5) Test Plan & 14 \\
\hline 3 & $5 / 22 / 2019$ & 5/29/2019 Issue ASNF (Task 5) Test Plan & 7 \\
\hline 4 & $5 / 29 / 2019$ & 8/21/2019 'Design Engineering Scale Experiment & 84 \\
\hline 5 & $7 / 31 / 2019$ & 8/21/2019 Issue Experiment Design Document & 21 \\
\hline 6 & $8 / 21 / 2019$ & 11/13/2019 'Fabrication and Installation of Engineering Scale Test Systems & 84 \\
\hline 7 & $8 / 21 / 2019$ & 10/23/2019 Identification of Functional Operability Criteria & 63 \\
\hline 8 & $10 / 23 / 2019$ & 11/13/2019 Issue System Operability Test Plans & 21 \\
\hline 9 & $10 / 30 / 2019$ & 11/13/2019 Acceptance of Fabricated Items & 14 \\
\hline 10 & $11 / 13 / 2019$ & 12/18/2019 'Performance of System Operability Testing & 35 \\
\hline 11 & $11 / 27 / 2019$ & 12/18/2019 Acceptance of System Operability Test Results & 21 \\
\hline 12 & $12 / 18 / 2019$ & 1/8/2020 "Optimization Test Runs & 21 \\
\hline 13 & $12 / 25 / 2019$ & 1/8/2020 Transmittal of Optimization Data & 14 \\
\hline 14 & $1 / 8 / 2020$ & 4/1/2020 Experimental Tests & 84 \\
\hline 15 & $3 / 18 / 2020$ & 4/1/2020 Transmittal of Experimental Test Data & 14 \\
\hline 16 & $11 / 13 / 2019$ & 4/1/2020 Analysis of Samples & 140 \\
\hline 17 & $11 / 13 / 2019$ & 4/1/2020 Transmittal of Analytical Results & 140 \\
\hline 18 & $5 / 29 / 2019$ & 3/24/2020 Model Development, Validation, and Updates & 300 \\
\hline 19 & $3 / 3 / 2020$ & $3 / 24 / 2020$ Integration of Drying Models into Dry Storage Models & 21 \\
\hline 20 & $4 / 1 / 2020$ & 5/6/2020 'Final Project Report Integration and Editing & 35 \\
\hline 21 & $5 / 6 / 2020$ & 5/13/2020 Agreement on Any Limitations on Distribution & 7 \\
\hline 22 & $5 / 13 / 2020$ & $5 / 13 / 2020$ End & 0 \\
\hline
\end{tabular}


ASNF Task 5 Experiment Test Plan

Revision: 1

Effective Date: 8/15/2019

Figure 1. Schematic Timeline for Deliverables (and supporting activities)

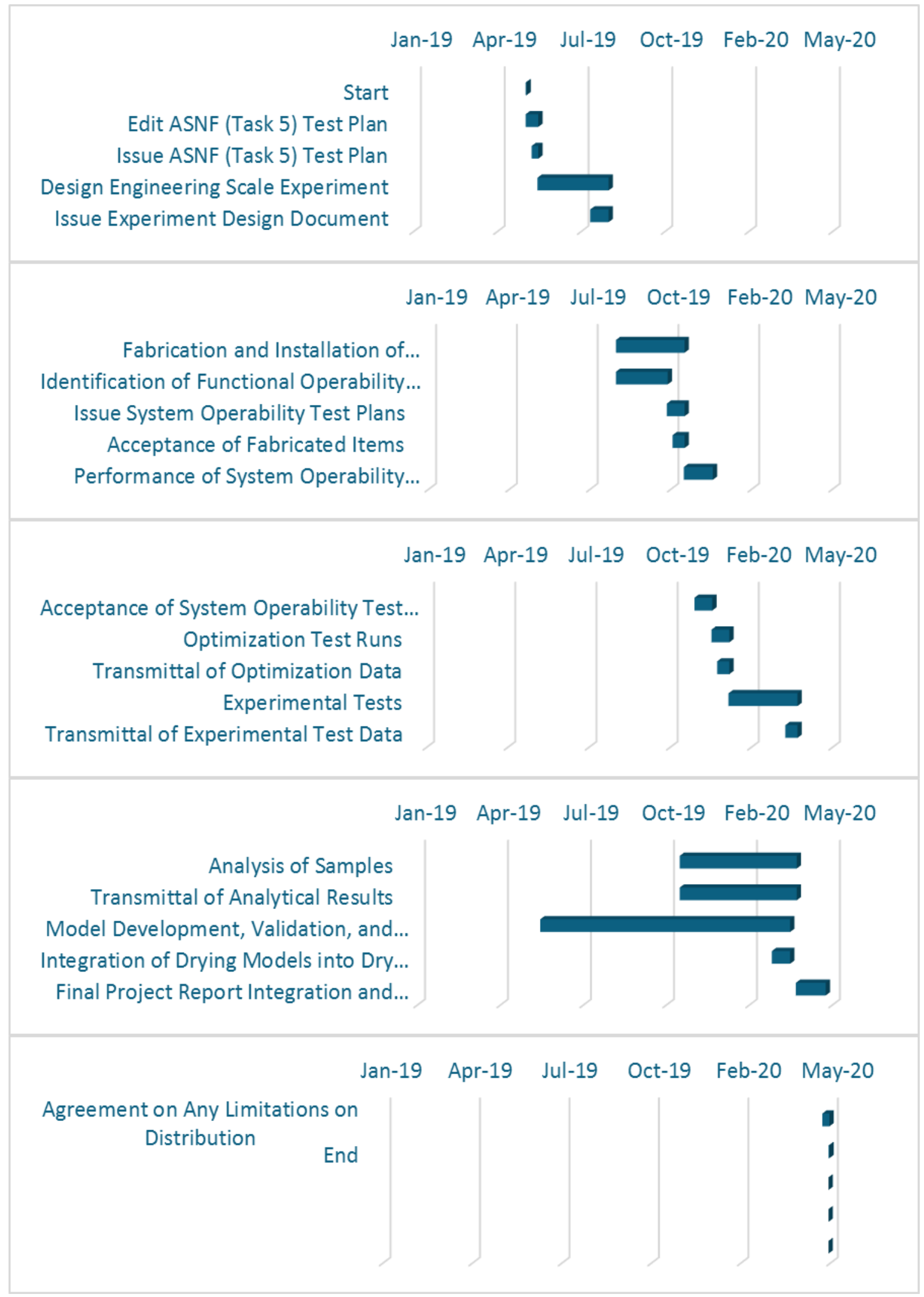




\section{REFERENCES}

1. Connolly, M. J., Aluminum Clad Spent Nuclear Fuel Long Term Dry Storage Technical Issues Action Plan - Technical and Engineering Activities, INL/EXT-17-43908, 2017.

2. Glazoff, M. V., and T. E. Lister, Transition of Spent Nuclear Fuel to Dry Storage: Modeling activities concerning aluminum spent nuclear fuel cladding integrity, INL/EXT-18-51694, Rev. 0, December 2018.

3. Metzger, K. E., R. E. Fuentes, A. L. d'Entremont, L. C. Olson, and R. L. Sindelar, Preparation of Aluminum Oxide Films Under Water Exposure - Preliminary Report on 1100 Series Alloys, SRNL-STI-2018-00427, Rev. 0, August 2018.

4. d'Entremont, A. L., R. E. Fuentes, L. C. Olson, and R. L. Sindelar, Preparation of Aluminum Oxide Films Under Water Exposure - Preliminary Report on 6061 Series Alloys, SRNL-STI2018-00449, Rev. 0, August 2018.

5. d'Entrement, A. L., R. E. Fuentes, L. C. Olson, and R. L. Sindelar, Preparation of Aluminum Oxide Films Under Water Exposure - Preliminary Report on 5052 Series Alloys, SRNL-STI2018-00646, Rev. 0, November 2018.

6. Lister, T., Vapor Phase Corrosion Testing of Pretreated AA 1100, INL/EXT-18-52249, December 2018.

7. NACE, National Association of Corrosion Engineers, Corrosion Basics, ISBN 0-915567-02-4, 1984.

8. Wefers, K., and C. Misra, Oxides and Hydroxides of Aluminum, Alcoa Technical Paper No. 19, Revised, Alcoa Laboratories, 1987.

9. Connolly, M. J., T. E. Bean, J. O. Brower, M. W. Patterson, A. K. Robb, R. Sindelar, R. E. Smith, V. E. Tonc, J. L. Tripp, and P. L. Winston, ATR Spent Fuel Management Options Study, INL/EXT-16-40471, January 2017. 